\title{
12-Step Programs to Reduce Illicit Drug Use
}

\author{
Sine Kirkegaard Nielsen, Trine Filges ${ }^{*}$ and Anne-Marie Klint Jørgensen
}

\author{
The National Centre for Social Research (SFI), Denmark
}

\begin{abstract}
Many treatments are not rigorously evaluated as to their effectiveness, and it is uncertain which types of interventions are more effective than others in reducing illicit drug use. The aim of this paper is to provide a systematic mapping of the research literature of the effectiveness of 12-step programs in reducing illicit drug use. A systematic literature search was conducted based on 17 international and Nordic bibliographic databases. A total of 15,993 references were screened, and eleven unique studies were finally included in this mapping. The included studies demonstrated conflicting results regarding the effectiveness of the 12-step treatment and TSF in reducing individuals' drug use. Two studies reported a positive effect of the TSF treatment compared to the comparison conditions in reducing drug use. Six studies reported no differences between the 12-step program and the comparison condition, and the remaining three studies reported a greater effect of the comparison condition. However, the included studies demonstrate that, in general, 12-step programs and the comparison conditions have some positive effects in reducing drug use and supporting abstinence among the participants.
\end{abstract}

Keywords: 12-step, TSF, illicit drugs, mapping, literature review.

\section{INTRODUCTION}

Illicit drug use ${ }^{1}$ remains a severe problem worldwide, and one that has serious costs for both individuals and societies in terms of social, health, and criminal problems (United Nations Office on Drugs and Crime 2013). The United Nations Office on Drugs and Crime (UNODC) estimated that in 2011, between 167 million and 315 million people globally between the age 15 and 64 had used an illicit substance ${ }^{2}$ at least once in the previous year (UNODC 2013). In light of the social consequences and significant financial costs of illicit drug use and criminality, there is a need to identify effective methods for reducing illicit drug use. However, many methods are not stringently evaluated as to their effectiveness, and it is unclear which types of interventions are more effective than others. The aim of this paper is to provide a systematic mapping of the research literature that evaluates 12-step programs targeting individuals with substance abuse. Although a large number of studies have examined the use of the approach, no systematic knowledge of the effectiveness of the 12-step programs is currently available. A Cochrane review has focused on 12-step programs and their effectiveness on alcohol dependence, but this review did not include dependence on illicit drugs (Ferri, Amato, and Davoli 2009).

*Address correspondence to this author at The National Centre for Social Research (SFI), Herluf Trolles Gade 11, Denmark-1052 København K., Denmark; Tel: +45 33480833; Fax: +45 33480800; E-mail: tif@sfi.dk

${ }^{1}$ In this paper, we reserve the term 'drug use' to apply to the illegal, nonmedical use of drugs.

${ }^{2}$ Illicit substances include opium/heroin (opiates), cocaine, cannabis and amphetamine-type stimulants (including MDMA/Ecstasy). 12-step approaches are one of the most internationally widespread treatments for drug use. Alcoholics Anonymous (AA) provides the oldest and most widely attended 12-step groups (Alcoholics Anonymous 2012; Mäkelä et al. 1996). The ideology of the approach is to offer individuals a new way of living which will support them in breaking the cycle of addiction and in maintaining abstinence (Mercer and Woody 1999). According to 12-step treatment approaches, individuals have lost control over their drug use, as a result of biological and/or psychological vulnerability. The 12-step treatment attempts to motivate the individuals into accepting the disease model of addiction (i.e. that addiction is a lifelong disease), and to believe in abstinence as the treatment goal. This includes involvement in different 12-step activities, for example, attending meetings, obtaining a sponsor, and working through the 12 steps in the program (Finney et al. 1998).

In this paper, we will include self-help groups working with the 12 steps, such as Narcotics Anonymous (NA) and Twelve Step Facilitation (TSF) treatments. The 12-step self-help groups typically work through specific principles, and the meetings usually follow a prescribed format that includes the 12-step readings. The main idea is that individuals progress toward recovery by going through "Twelve Steps", starting with an acknowledgement of the addiction, and ending with their participation in and ability to help others out of their addiction (Narcotics Anonymous 2008). The participation in the 12-step self-help groups is voluntary, and the treatment is guided by recovering individuals rather than professional therapists. The sponsorship is another important component; this is where a member who has made progress toward 
recovery shares his or her experience with another member of the 12-step group. In the 12-step program of NA, the steps contain a strong spiritual emphasis and encourage members to embrace spiritual values (Narcotics Anonymous 2008).

The 12-step approach is also used in Twelve Step Facilitation (TSF) treatments (Project Match Research Group 1997; Galanter and Kleber 2008). TSF treatments differ from self-help groups because the TSF treatments are typically organized around a treatment facilitation center, where the treatment is managed by professional therapists. The Minnesota model is one of the best known TSF treatments, and is characterized by the use of the 12-step philosophy as a foundation for therapeutic change. Individuals will usually participate in 12-steps meetings (for example NA) after completing TSF treatment. The TSF treatment can be delivered in both in-patient and outpatient settings.

\section{METHODOLOGY}

\subsection{Selection Criteria}

Selection criteria for which studies to include in this mapping fall into the following categories; types of studies, types of participants, types of intervention, and types of outcomes.

\subsubsection{Type of Studies}

The studies needed to be randomized controlled trials, quasi-randomized controlled trials $^{3}$ or quasiexperimental studies ${ }^{4}$ to be eligible for inclusion. The inclusion of quasi-randomized trials and quasiexperimental studies was necessary because of the open-door membership policy of the 12-step programs. This is especially pertinent to the self-help groups, and complicates the assessment of the effectiveness of treatments using a formal randomized controlled trial.

\subsubsection{Participants}

The population in the included studies consists of individuals who used illicit drugs, regardless of age, gender or ethnic background. We also included individuals with more than one type of drug use, for example, the combination of alcohol and cocaine.

\footnotetext{
${ }^{3}$ Where participants are allocated by, for example, alternate allocation, birth date, date of the week or month, case number or alphabetical order.

${ }^{4}$ Where pre-treatment group equivalence is demonstrated via for example matching, key risk variables, or where key risk variables are controlled for statistically.
}

\subsubsection{Intervention}

Studies needed to evaluate 12-step programs (in either the self-help or TSF format), delivered with the aim of absolute abstinence or a reduction in drug use, in order to be eligible for inclusion. Those 12-step programs that focus exclusively on treating alcohol dependence, such as AA programs, were excluded. Studies evaluating 12-step programs as an aftercare treatment were also excluded. Eligible control and comparison conditions were no intervention, waitlist controls, and any other intervention(s) not based on 12 step treatment philosophy or involvement in 12-step groups.

\subsubsection{Outcome}

The primary outcome needed to be abstinence or reduction of drug use, and we have only included studies that considered this outcome. Studies examining more than one type of drug use, for example, the combination of alcohol and cocaine, have been included if they report the outcomes separately. The outcomes can, for example, be measured by biochemical tests, estimates on drug use, and psychometric scales.

\subsection{Search Strategy}

The systematic literature searches were conducted in 2010-2013, and we used an extensive search strategy to identify the relevant studies. A wide range of electronic bibliographic databases ${ }^{5}$ were searched, along with government and policy databanks, a grey literature database, and citations in other reviews. In addition, we searched the reference list of included primary studies, hand searched relevant journals, and searched the internet using Google. We also corresponded with international experts to identify unpublished and on-going studies within the field of 12step. Neither date nor language restrictions were applied to the search. All retrieved hits were screened on title and abstract for relevance. Quantitative studies that evaluated a 12-step program were retrieved and screened in full-text to determine whether they were eligible for inclusion.

In the screening-process we focused on the categories; characteristics of the populations,

\footnotetext{
${ }^{5}$ The following bibliographic databases were searched: Medline, PsycINFO, Cinahl, Embase, Cochrane Library (including CENTRAL), Science Citation Index, SocINDEX, Social Care Online, ASSIA, Sociological Abstracts, SweMed+, Artikelsök, DiVA (Digitalevetenskapeligaarkivet), Bibliotek.dk, Bibsys, and Libris.
} 
Table 1: Description of Studies

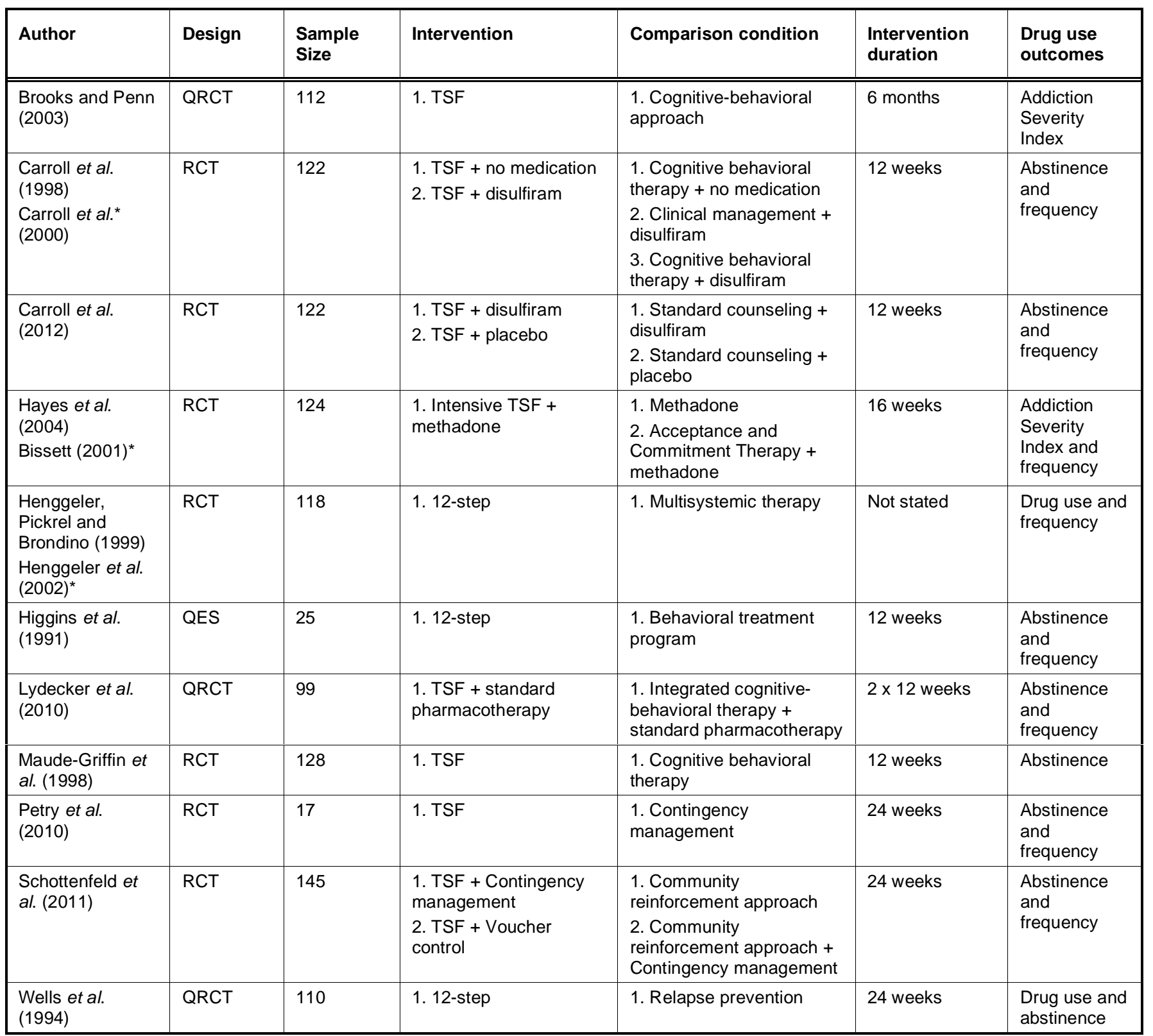

characteristics of the intervention and control/comparison condition, research design, sample size, outcomes, and results. We extracted and coded data in the web-based software program Eppi-reviewer.

\section{RESULTS}

\subsection{Results of the Search}

The systematic literature search yielded a total of 15,993 potential references. All 15,993 were screened on title and abstract and 347 records were retrieved and screened in full text. Of these, 313 did not meet the inclusion criteria and were excluded, and seven papers were not obtainable. Two papers (Donovan et al. 2013 and Majer et al. 2013) were excluded because the comparison condition were contaminated with 12-step principles or 12-step meetings. A total of 11 studies were finally data-extracted and included in this mapping.

\subsection{Description of the Included Studies}

Table 1 presents the 11 included studies (and the studies reporting on the same trials ${ }^{6}$ ) according to author, design, sample size, intervention, comparison

\footnotetext{
${ }^{6 *}$ The study is reporting on the same trial as the main study.
} 
condition, intervention duration and drug use outcomes.

The 11 studies were all performed in the US and cover different kinds of 12-step treatments using very diverse target populations, approaches to the intervention, comparison groups and outcome measures. Eight of the studies examine the Twelve Step Facilitation (TSF) treatment, while two of the studies investigate TSF treatment in combination with another intervention (standard pharmacotherapy, contingency management, medical treatment, voucher control). The remaining three studies meanwhile examine different kinds of 12-step programs, mainly self-help groups working with the principles of the12 steps in different ways.

Seven studies were described by the investigators as randomized controlled trials. Within these seven studies, four used an urn randomization procedure (Carroll et al. 2012; Petry et al. 2010; Schottenfeld et al. 2011). In the three remaining studies, the randomization procedure was not reported (Carroll et al. 1998; Hayes et al. 2004; Henggeler, Pickrel, and Brondino 1999; Maude-Griffin et al. 1998). Three studies were described as quasi-randomized controlled trials, but the randomization procedures were either unclear or not stated in the studies (Brooks and Penn 2003; Lydecker et al. 2010; Wells et al. 1994). The last study was a quasi-experimental study (Higgins et al. 1991). The unit of the randomization in the randomized controlled trials and the quasi-randomized controlled trials was individual, except Henggeler et al. (1999) who randomized by family. The sample size in the included studies generally varied from 100 to 170 participants, except for one study, which had a fairly small sample size of 25 participants (Higgins et al. 1991).

The included studies compared either 12-step treatment or TSF with different kinds of group-based and individual-oriented interventions. In general, all the included studies had different treatments as a comparison condition, but in some studies the comparison condition was combined with components from other interventions or with a medical treatment. The most frequently evaluated comparison condition was a different approach of behavioral therapy. Brooks and Penn (2003) compared TSF with an approach of Cognitive Behavioral Treatment (CBT), which consisted of self-management and recovery training (SMART). Maude-Griffin et al. (1998) evaluated the efficiency of CBT and TSF in treating cocaine abuse. Higgins et al.
(1991) assessed the efficiency of a behavioral treatment program (BTP) for achieving initial cocaine abstinence. Carroll et al. (1998) and Lydecker et al. (2010) both examined CBT combined with another treatment. Carroll et al. (1998) evaluated three comparison conditions, where the first condition was CBT alone, the second was CBT combined with disulfiram, and the third was contingency management only. Lydecker et al. (2010) evaluated integrated CBT combined with a standard pharmacotherapy. Wells et al. (1994) studied a cognitive-behavioral relapse prevention (RP). Hayes et al. (2004) examined a different approach of behavioral therapy defined as the Acceptance and Commitment Therapy (ACT) in combination with methadone maintenance, and this study also evaluated the efficiency of methadone maintenance alone. The remaining four studies evaluated comparison conditions different from the CBT, but Henggeler et al. (1999) studied a Multisystemic Therapy (MST), which emerged from and has similarities with behavioral therapy. Carroll et al. (2012) studied a standard counseling approach in combination with disulfiram and standard counseling plus placebo in the context of a community-based methadone program. Petry et al. (2010) evaluated effects of a group-based Contingency Management intervention (CM). Schottenfeld et al. (2011) also examined a CM condition, but in combination with a Community Reinforcement Approach (CRA), while Schottenfeld et al. (2011) also evaluated the CRA in combination with voucher control ${ }^{7}$.

\subsubsection{Inclusion Criteria in Included Studies}

Criteria for participation varied significantly between the 11 studies. Eligibility for study participation differed mainly according to dependence, type of drug use, mental illness, and medical condition. In seven studies, one inclusion criterion was that the participants' drug use needed to be cocaine (Carroll et al. 1998; Carroll et al. 2012; Higgins et al. 1991; Maude-Griffin et al. 1998; Schottenfeld et al. 2011; Petry et al. 2010; Wells et al. 1994). Within these studies, Carroll et al. (2012) reported that inclusion was dependent upon cocaine being used in combination with alcohol, while Petry et al. (2010) specified that participants needed to have used either cocaine or opioids within the past year.

The remaining five studies indicated that participants with any kind of abuse or dependence

${ }^{7}$ Monetary vouchers provided contingent on cocaine-negative urine tests or noncontigency but yoked in value. 
were eligible and could be included in the study (Brooks and Penn 2003; Henggeler et al. 1999; Lydecker et al. 2010; Hays et al. 2004). Hayes et al. (2004) specified however that participants needed to be using two drugs concurrently and also be on met had one maintenance treatment (Hays et al. 2004).

Participants in the included studies were predominately males, with only one study reporting solely on females (Schottenfeld et al. 2011). Two studies focused exclusively on participants who were at least 18 years of age (Higgins et al. 1991; Wells et al. 1994), and one study included adolescents between the ages of 12-17 years only (Henggeler et al. 1999). The remaining eight studies did not have any inclusion criteria relating to age.

Another criterion for inclusion was associated with the participants' mental health. In two studies participants were required to have been diagnosed with mental illness to be eligible for the study (Brooks and Penn, 2003; Lydecker et al. 2010). Brooks and Penn (2003) stated that participants needed to have a serious mental illness. This included individuals with diagnoses such as schizophrenia, bipolar disorder, schizoaffective disorder, and major depression. Lydecker et al. (2010) solely included individuals with a diagnosis of lifetime major depressive disorder.

Furthermore, four of the studies had specific criteria for participation. Petry et al. (2010) solely included HIVpositive individuals who were members of an HIV dropin center. Schottenfeld et al. (2011) included only pregnant females or females who had custody of a young child. Henggeler et al. (1999) stated that participants needed to be on formal or informal probationary status, living with at least one parent figure, and to be resident in Charleston County, USA. Wells et al. (1994) included only participants who met the following specified criteria: continued use despite persistent or recurrent problems or recurrent use in physically hazardous situations, with symptoms lasting one month or longer or occurring repeatedly over a longer period of time.

\subsubsection{Exclusion Criteria in Included Studies}

Eight studies excluded participants with severe physical or mental illness, for example, individuals with dementia, pathological gambling problems, bipolar disorder, schizophrenia, psychotic disorder, or individuals with a high risk of suicide (Carroll et al. 1998; Carroll et al. 2012; Hayes et al. 2004; Higgins et al. 1991; Lydecker et al. 2010; Maude-Griffin et al.
1998; Schottenfeld et al. 2011; Petry et al. 2010; Wells et al. 1994). Within these studies, Carroll et al. (1998); Carroll et al. (2012); Higgins et al. (1991) and MaudeGriffin et al. (1998) also excluded participants with a current medical condition. Carroll et al. (1998) also excluded individuals on probation or parole, and Hayes et al. (2004) excluded individuals with imminent criminal justice proceedings. Lydecker et al. (2010) specified that they had excluded homeless individuals or individuals who lived too far from the study site.

The remaining two studies had more unique criteria for exclusion. Brooks and Penn (2003) stated that participants found by a psychiatrist to have an Axis $\|^{8}$ personality disorder diagnosis but who did not also have an Axis I diagnosis, were excluded from participation. Henggeler et al. (1999) excluded participants who had a sibling involved in the study, or if the individuals were already formally enrolled on another substance abuse treatment.

\subsection{Effects of the 12-Step Treatments}

This section describes the main findings in the included studies focusing on the effects of different approaches of 12-step treatment in reducing participants' drug use. The included studies generally report different and conflicting results associated with the efficiency of the 12-step treatment or the TSF treatment as compared to a group of other treatments.

Two studies favor TSF treatment compared to the comparison conditions (Carroll et al. 1998; Carroll et al. 2012). Carroll et al. (1998) compared TSF plus disulfiram and TSF plus no medication with the following three comparison conditions; CBT plus no medication, $\mathrm{CM}$ plus disulfiram, and CBT plus disulfiram. All five interventions were effective in reducing the participants' drug use, but there were no significant differences in the effectiveness between TSF and CBT. These two types of psychotherapies were both more effective than $\mathrm{CM}$ in fostering longer periods of consecutive abstinence from cocaine and abstinence from both cocaine and alcohol simultaneously, as well as a higher percentage of cocaine-free participants with greater reductions in use across time. In comparison, neither CBT nor TSF, when compared with $\mathrm{CM}$, were associated with significant reductions in drug use, as indicated by urine

${ }^{8}$ Current diagnosis was determined using the diagnostic evaluation from the referring treatment team psychiatrist and the results of the Psychiatric Diagnostic Inventory-R, the Mini Mental Status Exam, and a clinical interview. 
specimens. Carroll et al. (2012) compared TSF plus disulfiram and TSF plus placebo with standard counseling plus disulfiram and standard counseling plus placebo. The study reported that assignment to TSF treatment was associated with reduced cocaine use via self-report, and data from the one year followup indicated stable effects overall, with no significant differences by group. The authors concluded that TSF appears to be a feasible treatment associated with a modest reduction in drug use.

Six of the included studies report no different effects of reduction of drug use between 12-step treatment or TSF treatment and the comparison conditions (Brooks and Penn, 2003; Hayes et al. 2004; Henggeler et al. 1999; Petry et al. 2010; Schottenfeld et al. 2011; Wells et al. 1994).

Brooks and Penn (2003) concluded that both TSF and CBT had positive effects on drug use among dually diagnosed individuals, with neither of the interventions found to be superior across multiple outcome dimensions. Hayes et al. (2004) suggests that both intensive TSF and ACT may add to the benefits of methadone maintenance in the reduction of drug use in polysubstance-abusing opiate addicts. The study showed no significant difference in effects between intensive TSF treatment and methadone treatment compared to methadone treatment alone in reducing drug use. In the comparison of intensive TSF and methadone treatment with ACT and methadone, no significant conclusion can be drawn because of missing data. Henggeler et al. (1999) evaluated a 12-step approach compared to MST in the reduction of drug use to treatment. The results show no significant between-group differences for either marijuana use or cocaine use during treatment or at a four-year followup.

Petry et al. (2010) did not find any significant differences in drug use between participants in TSF or $\mathrm{CM}$ condition. The authors found that participants in the CM condition submitted a greater number of consecutive drug-free specimens than the participants in the TSF condition, but that the proportions of negative samples did not differ between the two groups during treatment or at one-year follow-up. Schottenfeld et al. (2011) demonstrated that both TSF paired with $\mathrm{CM}$ and TSF paired with a voucher control improved abstinence among pregnant women and women with young children, but the study did not support greater efficiency of TSF paired with CM or TSF paired with voucher control compared to the CRA condition or the
CRA paired with CM. Wells et al. (1994) showed that both the 12-step treatment and the RP reduced the participants' cocaine and marijuana use, but there were no differential effects of treatment type on cocaine and marijuana use over time.

The remaining three studies report a greater effect of the comparison conditions compared to the 12-step treatment or TSF treatment. Higgins et al. (1991) demonstrated that participants given the BTP achieved significantly longer periods of continuous abstinence than participants given the 12-step treatment. The main drug in the study was cocaine, and the two groups did not differ in other drug use analyzed as a function of treatment weeks. However, when collapsed across treatment weeks, the vast majority of the test results were negative in both groups, but the group given BTP had a significantly lower proportion of marijuananegative specimens than the 12 -step participants.

Lydecker et al. (2010) showed that CBT plus standard pharmacotherapy was associated with superior substance use outcomes (i.e. less substance use) compared with TSF plus standard pharmacotherapy 18 months after treatment entry. Participants in the CBT plus standard pharmacotherapy had greater reductions in frequency of drug and alcohol use over time compared with TSF plus standard pharmacotherapy. Maude-Griffin et al. (1998) also found the comparison condition to be more effective than TSF. Across two different outcome variables, the study showed that participants in CBT were significantly more likely to achieve abstinence than participants in TSF.

\section{CONCLUSION}

The systematic mapping of the research literature identified a wide range of research literature examining the effectiveness of the different 12-step programs. A total of 11 papers met the inclusion criteria and were included in the mapping. The 11 studies were all conducted in the US, and covered different approaches of the 12-step program with very diverse target populations, comparison conditions, and outcome measures. The inclusion and exclusion criteria in the studies varied significantly, and some studies had very specified criteria for participation. The target groups in the studies were also very diverse, but had some general characteristics according to gender, age, drug use, and mental health. The included studies compared either 12-step treatment or TSF with different kinds of group-based and individual-oriented interventions, and 
in some studies the comparison condition was paired with components from other interventions or with a medical treatment. The most frequently evaluated comparison condition was a different approach of behavioral therapy.

Only two studies found TSF to be more effective than the comparison conditions. Six studies reported no different effects of reduction in drug use between the 12-step program and the comparison conditions, while the remaining three studies reported a greater effect of the comparison conditions compared to the 12-step programs. The main findings in the included studies demonstrated a generally positive effect of both the 12-step programs and the comparison conditions in reducing participants' drug use and support abstinence. Overall, findings from this mapping did not point to significant differences between 12-step programs and the comparison conditions, but suggested instead that despite the theoretical differences between the interventions, use of either of these treatments is likely to benefit different groups of individuals with a substance abuse.

This paper has a couple of limitations. First, we did not report results regarding the magnitude of effect, as not all the included studies provided data suitable for effect size analysis. A quantitative data synthesis would thus leave out some of the studies. Secondly, we did not rigorously evaluate the quality of the studies. However, we defined different selection criteria and only included studies that met these criteria regarding study design, participants, intervention, and outcomes. In further research we will perform an in-depth assessment of the quality of the studies and pool the suitable outcome measures in a quantitative data synthesis.

\section{REFERENCES}

AlcoholicsAnonymous. 2012. Retrieved February 28, 2012, from www.aa.org

Bissett, Richard T. 2001. "Processes of change: Acceptance versus 12-Step in polysubstance-abusing methadone Clients." Reno: University of Nevada.

Brooks, Audrey J. and Patricia E. Penn. 2003. "Comparing Treatments for Dual Diagnosis: Twelve-Step and SelfManagement and Recovery Training." The American Journal of Drug and Alcohol Abuse 29(2):359-383. http://dx.doi.org/10.1081/ada-120020519

Carroll, Kathleen M., Charla Nich, Samuel A. Ball, Elinore McCance, and Bruce J. Rounsavile. 1998. "Treatment of Cocaine and Alcohol Dependence with Psychotherapy and Disulfiram." Addiction 93(5):713-727. http://dx.doi.org/10.1046/j.1360-0443.1998.9357137.x

Carroll, Kathleen M., Charla Nich, Samuel A. Ball, Elinore McCance, Tami L. Frankforter, and Bruce J. Rounsavile. 1998. "One- year follow-up of disulfiram and psychotherapy for cocainealcohol users: sustained effects of treatment." Addiction 95(9):1335-1349.

http://dx.doi.org/10.1046/j.1360-0443.2000.95913355.x

Carroll, Kathleen M., Charla Nich, Julia M. Shi, Dorothy Eagan, and Samuel A. Ball. 2012. "Efficacy of Disulfiram and Twelve Step Facilitation in Cocaine-Dependent Individuals Maintained on Methadone: A Randomized PlaceboControlled Trial." Drug and Alcohol Dependence 126(12):224-231.

http://dx.doi.org/10.1016/j.drugalcdep.2012.05.019

Donovan, Dennis T., Dennis C. Daley, Gregory S. Brigham, Candace C. Hodgkins, Harold I. Perl, Sharon B. Garrett, Suzanne R. Doyle, Anthony S. Floyd, Patricia C. Knox, Christopher Botero, Thomas M. Kelly, Therese K. Killeen, Carole Hayes, Nicole Kua'iBaumhofer, Cindy Seamans, and Lucy Zammarelli. 2013. "Stimulant abuser groups to engage in 12Step: A multisite trial in the National Institute on Drug Abuse Clinical Trials Network." Journal of Substance Abuse Treatment 44:103-114. http://dx.doi.org/10.1016/j.jsat.2012.04.004

Ferri, Marica, Laura Amato, and Marina Davoli. 2009. "Alcoholics Anonymous and other 12-step Programs for Alcohol Dependence." Cochrane Database of Systematic Reviews 2006, Issue 3. Art. No.: CD005032. http://dx.doi.org/10.1002/14651858.cd005032.pub2

Finney, John W., Charlotte A. Noyes, Adam I. Coutts, and Rudolf $\mathrm{H}$. Moos.1998."Evaluating Substance Abuse Treatment Process Models: I. Changes on Proximal Outcome Variables during 12-Step and Cognitive-Behavioral Treatment." Journal of Studies on Alcohol 59:371-380.

Galanter, Marc and Herbert D. Kleber. 2008. The American Psychiatric Publishing Textbook of Substance Abuse Treatment. $4^{\text {th }}$ ed. Washington, DC: American Psychiatric Publishing. Retrieved December 20, 2013 from http://psychiatryonline.org/book.aspx?bookid=26

Hayes, Steven C., Kelly G. Wilson, Elizabeth V. Gilford, Richard Bisset, Melissa Piasecki, Sonja V. Batten, Michelle Byrd, and Jennifer Gregg. 2004. "A Preliminary Trial of Twelve-Step Facilitation and Acceptance and Commitment Therapy With Polysubstance-Abusing Methadone-Maintained Opiate Addicts." Behavior Therapy 35:667-688. http://dx.doi.org/10.1016/s0005-7894(04)80014-5

Henggeler, Scott W., Susan G. Pickrel, and Michael J. Brondino. 1999. "Multisystemic Treatment of Substance-Abusing and Dependent Delinquents: Outcomes, Treatment Fidelity, and Transportability." Mental Health Services Research 1(3):171184.

http://dx.doi:10.1023/A:1022373813261

Henggeler, Scott W., William G. Glingempeel, Michael J. Brondino, and Susan G. Pickrel. 2002. "Four-Year Follow-up of Multisystemic Therapy With Substance-Abusing and Substance-Dependent Juvenile Offenders." Journal of the American Academy of Child and Adolescent Psychiatry 41(7):868-874. http://dx.doi:10.1097/00004583-200207000-00021

Higgins, Stephen T., Dawn D. Delaney, Alan J. Budney, and Warren K. Bickel. 1991. "A Behavioral Approach to Achieving Initial Cocaine Abstinence." The American Journal of Psychiatry 148(9):1218-1224.

Lydecker, Katherine P., Susan R. Tate, Kevin M. Cummins, John McQuaid, Eric Granholm, and Sandra A. Brown. 2010. "Clinical Outcomes of An Integrated Treatment for Depression and Substance use Disorders." Psychology of Addictive Behaviors 24(3):453-465. http://dx.doi.org/10.1037/a0019943

Majer, John M., Leonard A. Jason, Darrin M. Aase, Jocelyn R. Droege, Joseph R. Ferrari. 2013. "Categorical 12-step involvement and continuous abstinence at 2 years." Journal of Substance Abuse Treatment 44:46-51. http://dx.doi.org/10.1016/j.jsat.2012.03.001 
Maude-Griffin, Peg M., Jill M. Hohenstein, Gary L. Humfleet, Patrick M. Reilly, Donald J. Tusel, and Sharon M. Hall. 1998. "Superior Efficacy of Cognitive-Behavioral Therapy for Urban Crack Cocaine Abusers: Main and Matching Effects." Journal of Consulting and Clinical Psychology 66(5):832-837. http://dx.doi.org/10.1037/0022-006x.66.5.832

Mercer, Delinda E. and George E. Woody.1999. "Contributions of the 12-Step Approach". In An Individual Drug Counselling Approach to Treat Cocaine Addiction. Rockville, MD: National Institute on Drug Abuse, Division of Clinical and Services Research. http://archives.drugabuse.gov/ TXManuals/IDCA/IDCA3.html\#Contributions

Mäkelä, Klaus, likka Arminen, Kim Bloomfield, Irmgard EisenbachStangl, Karin Hermersson Bergmark, Noriko Kurube, Nicoletta Mariolini, and Antoni Zielinksi, eds. 1996. Alcoholics Anonymous as a Mutual-Help Movement: A study in Eight Societies. Madison, WI: Wisconsin University Press,

Narcotics Anonymous. 2008. Narcotics Anonymous: basic text. Sixth ed. Chatsworth, CA: Narcotics Anonymous World Services, Inc.

Petry, Nancy M., Jeremiah Weinstock, Sheila M. Alessi, Marilyn W. Lewis, and Kevin Dieckhaus. 2010. "Group-Based Randomized Trial of Contingencies for Health and
Abstinence in HIV Patients." Journal of Consulting and Clinical Psychology 78(1):89-97.

http://dx.doi.org/10.1037/a0016778

Project Match Research Group. 1997." Matching Alcoholism Treatments to Client Heterogeneity: Project MATCH Posttreatment Drinking Outcomes." Journal of Studies on Alcohol and Drugs, 58(1):7-29.

Schottenfeld, Richard S., Brent Moore, and Michael V. Pantalon. 2011. "Contingency Management with Community Reinforcement Approach or Twelve-Step Facilitation Drug Counseling for Cocaine Dependent Pregnant Women or Women with Young Children." Drug and Alcohol Dependence 118(1):48-55. http://dx.doi.org/10.1016/j.drugalcdep.2011.02.019

United Nations Office on Drugs and Crime, UNODC. 2013.World Drug Report 2013. New York, NY: United Nations Publication. http://www.unodc.org/wdr/

Wells, Elizabeth A., Peggy Peterson, Randy R. Gainey, David J. Hawkins, and Richard F. Catalano.1994. "Outpatient Treatment for Cocaine Abuse: A Controlled Comparison of Relapse Prevention and Twelve-Step Approaches." American Journal of Drug and Alcohol Abuse 20(1):1-17. http://dx.doi.org/10.3109/00952999409084053

\section{DOI: http://dx.doi.org/10.6000/1929-4409.2014.03.08}

(C) 2014 Nielsen et al.; Licensee Lifescience Global.

This is an open access article licensed under the terms of the Creative Commons Attribution Non-Commercial License (http://creativecommons.org/licenses/by-nc/3.0/) which permits unrestricted, non-commercial use, distribution and reproduction in any medium, provided the work is properly cited. 